\title{
Orbital ordering and exchange interaction in the manganites
}

\author{
Hakim Meskine, Harald König,* and Sashi Satpathy \\ Department of Physics and Astronomy, University of Missouri-Columbia, Columbia, Missouri 65211
}

(Received 30 October 2000; revised manuscript received 9 February 2001; published 15 August 2001)

\begin{abstract}
The microscopic origin of the exchange interaction in manganites is studied by solving an electronic model Hamiltonian for the Mn-O-Mn triad. It is shown that the magnetic structure of $\mathrm{La}_{1-x} \mathrm{Ca}_{x} \mathrm{MnO}_{3}$ is correctly described within an electronic Hamiltonian model, provided that the appropriate orientation of the $\operatorname{Mn}\left(e_{g}\right)$ orbitals induced by the Jahn-Teller effect is taken into account. The Jahn-Teller distortions of the $\mathrm{MnO}_{6}$ octahedra control the orientation of the $e_{g}$ orbitals in the crystal, which in turn is shown to determine the sign of the magnetic exchange. Electron hopping involving the $\operatorname{Mn}\left(t_{2 g}\right)$ orbitals is found to be important in certain situations, for instance, it can cause a sign change in the exchange interaction, from ferromagnetic to antiferromagnetic, as a function of the $\mathrm{Mn}-\mathrm{O}-\mathrm{Mn}$ bond angle. All our results are obtained by exact diagonalization of the model Hamiltonian, either by direct diagonalization or by diagonalization using the Lanczos method, if the Hamiltonian is too big, and are rationalized using results of the fourth-order perturbation theory. The exchange interactions (signs and magnitudes) of the end members $\mathrm{LaMnO}_{3}$ and $\mathrm{CaMnO}_{3}$ as well as of the half-doped compound, $\mathrm{La}_{1 / 2} \mathrm{Ca}_{1 / 2} \mathrm{MnO}_{3}$, are all described correctly within the model.
\end{abstract}

DOI: 10.1103/PhysRevB.64.094433

PACS number(s): 75.30.Et, 75.30.Vn, 72.80.Ga

\section{INTRODUCTION}

The magnetic phase diagram of the colossal magnetoresistive manganites $\mathrm{La}_{1-x} \mathrm{Ca}_{x} \mathrm{MnO}_{3}$ is quite rich because of the interplay between the charge, orbital, lattice, and spin degrees of freedom. A clear understanding of the coupling between these degrees of freedom is critical to the understanding of many of the intriguing properties of these materials including the colossal magnetoresistance.

This paper deals with the microscopic origin of the exchange interaction in the manganites. In the 1950s a set of semiempirical rules were formulated by Goodenough, Kanamori, and Anderson (GKA rules) to explain the magnetic interactions in manganites. ${ }^{1-4}$ More quantitative calculations of the magnitudes of the exchange were attempted only recently for $\mathrm{LaMnO}_{3}$ using first-principles electronic structure methods. ${ }^{5,6}$ The two calculations, one by Solovyev et al. ${ }^{5}$ employing the density-functional method and the other by $\mathrm{Su}$ et $a .^{6}{ }^{6}$ employing the $a b$ initio Hartree-Fock method, produced conflicting results even for the signs of the exchange interactions. The interplane $\mathrm{Mn}-\mathrm{O}-\mathrm{Mn}$ exchange for $\mathrm{LaMnO}_{3}$ obtained in Ref. 5 is in fact ferromagnetic in direct contradiction with the GKA rules. In view of this, it is important to examine the magnetic exchange in manganites.

The approach in this paper is to understand the origin of the magnetic exchange from the solution of a simple, minimal model. The Hamiltonian model is simple enough that it can be solved exactly. The needed electronic parameters are taken with guidance from the $a b$ initio density-functional results. ${ }^{7-9}$

It is shown that within an electronic Hamiltonian model, the magnetic exchange in the $\mathrm{La}_{1-x} \mathrm{Ca}_{x} \mathrm{MnO}_{3}$ system can be described correctly, if one takes into account the appropriate Jahn-Teller splitting of the $e_{g}$ orbitals induced by the coupling to the $\mathrm{MnO}_{6}$ octahedra. The model not only describes correctly the exchange interactions in $\mathrm{LaMnO}_{3}$, but also in the other two members in the $\mathrm{La}_{1-x} \mathrm{Ca}_{x} \mathrm{MnO}_{3}$ series, viz., $\mathrm{CaMnO}_{3}$ and $\mathrm{La}_{1 / 2} \mathrm{Ca}_{1 / 2} \mathrm{MnO}_{3}$. Thus the model correctly describes the type- $A$, type- $G$, and type-CE magnetic structures (Fig. 1) observed in $\mathrm{LaMnO}_{3}, \mathrm{CaMnO}_{3}$, and $\mathrm{La}_{1 / 2} \mathrm{Ca}_{1 / 2} \mathrm{MnO}_{3}$, respectively. ${ }^{10-12}$ In addition, we show that the electron hopping involving the $\operatorname{Mn}\left(t_{2 g}\right)$ orbitals adds a net ferromagnetic component and in certain situations it re-
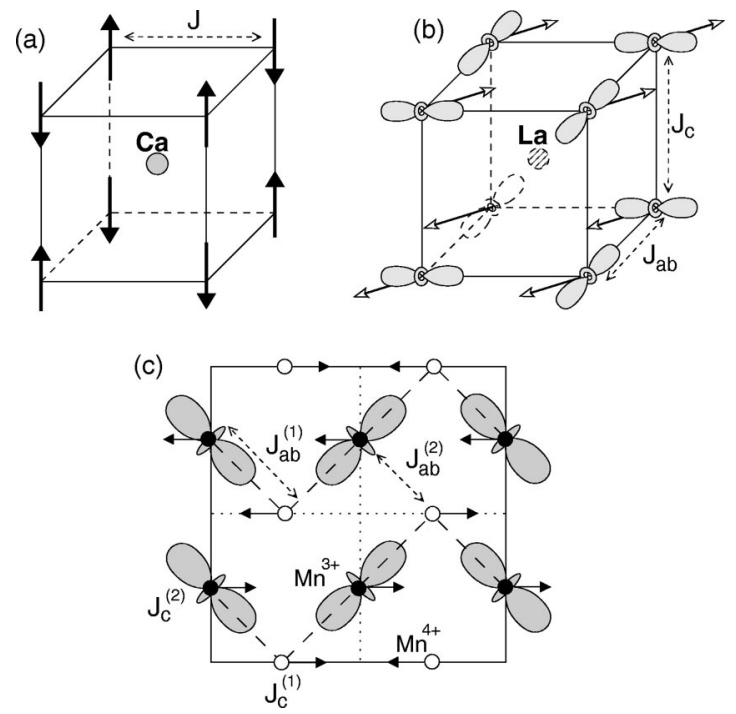

FIG. 1. Magnetic and orbital ordering in the type- $G$ (a), type- $A$ (b), and type-CE (c) structures observed in $\mathrm{CaMnO}_{3}, \mathrm{LaMnO}_{3}$, and $\mathrm{La}_{1 / 2} \mathrm{Ca}_{1 / 2} \mathrm{MnO}_{3}$, respectively. In the CE structure, the basal $(a b)$ planes are stacked one over the other, along the $c$ direction with the Mn spins reversed on the successive planes. The dashed lines in (c) indicate the ferromagnetically coupled zigzag chains of $\mathrm{Mn}$ atoms. The $J$ 's indicate the magnetic exchange between the Mn atoms: $J_{a b}^{(1)}$ and $J_{a b}^{(2)}$ are the intraplanar exchange interactions, while $J_{c}^{(1)}$ and $J_{c}^{(2)}$ denote the interplanar interactions, along bonds perpendicular to the plane of paper. Arrows indicate magnetic moments on the $\mathrm{Mn}$ atoms. 


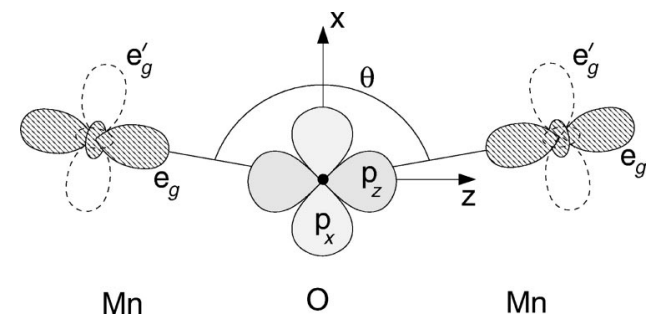

FIG. 2. The Mn-O-Mn triad considered in the paper and the nomenclature for the different orientations of the $\operatorname{Mn}\left(e_{g}\right)$ orbital. The $z^{2}-1$ orbital oriented along a Mn-O bond is referred to as the $e_{g}$ orbital, while the ones perpendicular to it are referred to as $e_{g}^{\prime}$ or $e_{g}^{\prime \prime}$. The $e_{g}^{\prime}$ orbital lies on the plane of the figure as shown while the $e_{g}^{\prime \prime}$ (not shown) lies perpendicular to the plane. The corresponding counterparts forming the two-dimensional manifold of the $\operatorname{Mn}\left(e_{g}\right)$ orbitals are denoted by $E_{g}, E_{g}^{\prime}$, or $E_{g}^{\prime \prime}$. Thus if $e_{g}=z^{2}-1$ in a certain coordinate system, then $E_{g}=x^{2}-y^{2}$ and so on. The $y$ axis is normal to the plane of the triad.

verses the sign of the exchange interaction as the Mn-O-Mn bond angle is varied.

\section{THE HAMILTONIAN AND ITS PARAMETERS}

Consider two $\mathrm{MnO}_{6}$ octahedra sharing a common vertex via an oxygen atom as is encountered in the manganites. The bond angle of the $\mathrm{Mn}-\mathrm{O}-\mathrm{Mn}$ triad is $180^{\circ}$ in the ideal perovskite structure but deviates somewhat from this ideal value in the actual crystal. Since the manganites are mixed valence systems consisting of $\mathrm{Mn}^{3+}$ and $\mathrm{Mn}^{4+}$ ions, with electronic configurations of $t_{2 g}^{3} e_{g}^{1}$ and $t_{2 g}^{3} e_{g}^{0}$, respectively, we can have any of the three combinations of $\mathrm{Mn}$ valence on the triad, viz., $\mathrm{Mn}^{3+}-\mathrm{O}-\mathrm{Mn}^{3+}, \mathrm{Mn}^{4+}-\mathrm{O}-\mathrm{Mn}^{4+}$, and $\mathrm{Mn}^{3+}-\mathrm{O}-\mathrm{Mn}^{4+}$. These are found in the three compounds $\mathrm{LaMnO}_{3}, \mathrm{CaMnO}_{3}$, and $\mathrm{La}_{1 / 2} \mathrm{Ca}_{1 / 2} \mathrm{MnO}_{3}$, respectively.

Now, the $\mathrm{Mn}^{3+}$ being a Jahn-Teller ion, the oxygen octahedron surrounding it is distorted, with the result that the degeneracy of the $e_{g}$ orbitals is removed. The orbital with the lower energy is a " $z^{2}-1$ "-type orbital with its lobes pointed towards the longest $\mathrm{Mn}-\mathrm{O}$ bond. This is sometimes referred to as the "long" cation-anion bond, while the rest of the $\mathrm{Mn}-\mathrm{O}$ bonds within the $\mathrm{MnO}_{6}$ octahedron are the "short" bonds. The orientation of the occupied " $z^{2}-1$ " orbital is along the $\mathrm{Mn}-\mathrm{O}$ bond if it is long, or else the orientation is perpendicular to it, if the bond is short.

The orientations of the $\mathrm{Mn}$ and the oxygen orbitals are illustrated in Fig. 2. The orbitals explicitly considered here are the three $\mathrm{O}(p)$ orbitals and the five $\mathrm{Mn}(d)$ orbitals. Because the $e_{g}$ orbitals are partially occupied in $\mathrm{Mn}^{3+}$ and since the electronic hoppings for the two orbitals are different, the magnetic exchange will depend on which of these two orbitals is occupied.

Keeping this in mind, we introduce the nomenclature of $e_{g}, e_{g}^{\prime}$, or $e_{g}^{\prime \prime}$, for the occupied $e_{g}$ orbital in $\mathrm{Mn}^{3+}$, depending on how it is oriented with respect to the $\mathrm{Mn}-\mathrm{O}$ bond in the triad as shown in Fig. 2. The $e_{g}$ orbital is a $z^{2}-1$-type orbital with the $z$ axis along the Mn-O bond. The $e_{g}^{\prime}$ is a similar $z^{2}-1$-type orbital except that now the $z$ axis is on the plane of the $\mathrm{Mn}-\mathrm{O}-\mathrm{Mn}$ triad and perpendicular to the $\mathrm{Mn}-\mathrm{O}$ bond. The $e_{g}^{\prime \prime}$ is oriented perpendicular to both $e_{g}$ and $e_{g}^{\prime}$ just defined. It is however not relevant to the present case since the $e_{g}^{\prime \prime}$ orbital orientation is not found in the compounds under study here. The remaining orbital of the two-dimensional manifold will be called $E_{g}, E_{g}^{\prime}$, or $E_{g}^{\prime \prime} . E_{g}$, for instance, denotes the $x^{2}-y^{2}$ orbital, with $z$ axis along the Mn- $\mathrm{O}$ bond. The $y$ axis is chosen to be normal to the plane of the triad.

It turns out that the orientation of these $e_{g}$ orbitals, in case of the $\mathrm{Mn}^{3+}$ atom, will have a direct bearing on the magnetic coupling. ${ }^{13,14}$ For this reason, even though the oxygen atoms forming the $\mathrm{MnO}_{6}$ octahedra around the $\mathrm{Mn}$ atoms are not explicitly considered in our Hamiltonian, the Jahn-Teller splittings of the $e_{g}$ orbitals induced by the distortions of the $\mathrm{MnO}_{6}$ octahedra are taken into account properly.

With these considerations, the Hamiltonian for the Mn$\mathrm{O}-\mathrm{Mn}$ triad is written as

$$
\mathcal{H}=\mathcal{H}_{\mathrm{KE}}+\mathcal{H}_{\text {Coulomb }}+\mathcal{H}_{\text {Hund }}
$$

where the three terms are, respectively, the kinetic, Coulomb, and the Hund's-rule energies,

$$
\begin{aligned}
& \mathcal{H}_{\mathrm{KE}}= \sum_{i \nu}^{\mathrm{O}} \epsilon_{p} n_{i \nu}+\sum_{i \alpha \nu}^{\mathrm{Mn}} \epsilon_{d}\left(\alpha, Q_{i}\right) n_{i \alpha \nu} \\
&+\sum_{\langle i j\rangle \alpha \beta \nu} t_{i \alpha, j \beta}\left(c_{i \alpha \nu}^{\dagger} c_{j \beta \nu}+\text { H.c. }\right), \\
& \mathcal{H}_{\text {Coulomb }}=\sum_{i}^{\mathrm{Mn}, \mathrm{O}} n_{i}\left(n_{i}-1\right) U_{i}
\end{aligned}
$$

and

$$
\mathcal{H}_{\text {Hund }}=-J_{H} \sum_{i \alpha}^{\text {Mn }} n_{i \alpha \uparrow} n_{i \alpha \downarrow} .
$$

Here, $i, \alpha, \nu$ are, respectively, the site (manganese or oxygen), orbital (the five $d$ orbitals on Mn and the three $p$ orbitals on oxygen), and spin indices. $\langle i j\rangle$ indicates summation over the nearest neighbors, $c_{i \alpha \nu}^{\dagger}$ is the creation operator, $n_{j \beta \nu}$ is the corresponding number operator, and the site occupancy $n_{i} \equiv \sum_{\alpha \nu} n_{i \alpha \nu}$.

The first term in the Hamiltonian is the kinetic energy term. The matrix elements $t_{i \alpha, j \beta}$ are the appropriate KosterSlater tight-binding hopping integrals ${ }^{15,16}$ between the Mn and the $\mathrm{O}$ atoms. As indicated from band calculations, $\mathrm{Mn}(d)-\mathrm{O}(p)$ hopping is an important hopping in the problem and only this has been retained in the Hamiltonian Eq. (1). The hopping integrals between different orbitals are listed in Table I in terms of the two $p$ - $d$ hopping parameters $V_{p d \sigma}$ and $V_{p d \pi}$. The second term $\mathcal{H}_{\text {Coulomb }}$ represents the on-site Coulomb interaction.

The last term is the Hund's-rule energy that favors parallel alignment of electron spins on the Mn site. The Hund's en$\operatorname{ergy} J_{H}$ is of the order of $1 \mathrm{eV}$ for the $\mathrm{Mn}$ atom, but is often taken to be $\infty$ for simplicity. Sometimes a simpler version of the Hund's-rule energy is used in the literature, 
TABLE I. Koster-Slater tight-binding matrix elements $\langle a|H| b\rangle$ between the oxygen $p$ orbitals and manganese $d$ orbitals. Here, $t$ $=V_{p d \sigma}, t^{\prime}=V_{p d \pi}, \alpha=\cos (\theta / 2)$ and $\beta=\sin (\theta / 2)$. The $e_{g}, e_{g}^{\prime}$, and $e_{g}^{\prime \prime}\left(E_{g}, E_{g}^{\prime}\right.$, and $\left.E_{g}^{\prime \prime}\right)$ orbitals have specific orbital orientation with respect to the Mn-O-Mn bond as indicated in Fig. 1 and the corresponding text.

\begin{tabular}{cccccc}
\hline \hline & $\left|e_{g}\right\rangle$ & $\left|E_{g}\right\rangle$ & $|x y\rangle$ & $|y z\rangle$ & $|z x\rangle$ \\
\hline$\langle x|$ & $-\alpha t$ & 0 & 0 & 0 & $-\beta t^{\prime}$ \\
$\langle y|$ & 0 & 0 & 0 & $t^{\prime}$ & 0 \\
$\langle z|$ & $-\beta t$ & 0 & 0 & 0 & $\alpha t^{\prime}$ \\
& $\left|e_{g}^{\prime}\right\rangle$ & $\left|E_{g}^{\prime}\right\rangle$ & $|x y\rangle$ & $|y z\rangle$ & $|z x\rangle$ \\
\hline$\langle x|$ & $-\frac{\alpha t}{2}$ & $\frac{\sqrt{3} \alpha t}{2}$ & 0 & 0 & $-\beta t^{\prime}$ \\
$\langle y|$ & 0 & 0 & $t^{\prime}$ & 0 & 0 \\
$\langle z|$ & $\frac{\beta t}{2}$ & $-\frac{\sqrt{3} \beta t}{2}$ & 0 & 0 & $-\alpha t^{\prime}$ \\
& $\left|e_{g}^{\prime \prime}\right\rangle$ & $\left|E_{g}^{\prime \prime}\right\rangle$ & $|x y\rangle$ & $|y z\rangle$ & $|z x\rangle$ \\
\hline$\langle x|$ & $-\frac{\alpha t}{2}$ & $\frac{\sqrt{3} \alpha t}{2}$ & $-\beta t^{\prime}$ & 0 & 0 \\
$\langle y|$ & 0 & 0 & 0 & 0 & $t^{\prime}$ \\
$\langle z|$ & $\frac{\beta t}{2}$ & $-\frac{\sqrt{3} \beta t}{2}$ & $-\alpha t^{\prime}$ & 0 & 0 \\
\hline \hline & 0 & & & 0 \\
\hline
\end{tabular}

$$
\mathcal{H}_{\text {Hund }}=-J_{H} \sum_{i}^{\text {Mn }} \vec{S}_{i} \cdot \vec{\sigma}_{i} \text {, }
$$

especially when the $\operatorname{Mn}\left(t_{2 g}\right)$ spins are treated as classical core spins $\vec{S}$ interacting with itinerant electrons (spin $\vec{\sigma}_{i}$ ) with no hopping allowed for the $t_{2 g}$ electrons. The earlier expression Eq. (4) for $\mathcal{H}_{\text {Hund }}$ allows us to treat the $t_{2 g}$ electrons as mobile with no fixed core spin. The $t_{2 g}$ hopping will be shown later to significantly contribute to the magnetic interaction in the manganites. Though not exactly identical, the two forms of the Hund's energy $\mathcal{H}_{\text {Hund }}$ are analogous and describe the same physics as far as manganites are concerned.

The on-site energies, indicated in Fig. 3 for the

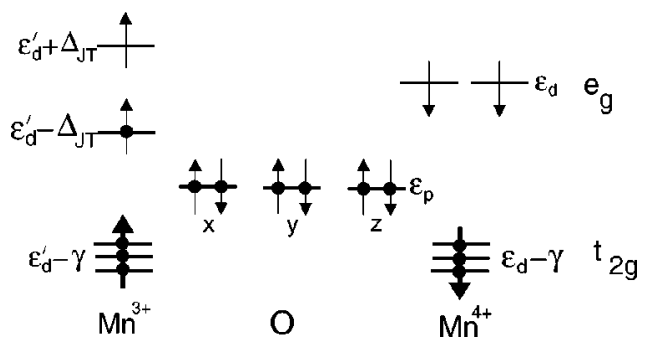

FIG. 3. Electronic configuration and one-electron orbital energies for $\mathrm{Mn}^{3+}-\mathrm{O}-\mathrm{Mn}^{4+}$. The Jahn-Teller splitting at the $\mathrm{Mn}^{3+}$ site is caused by the oxygen octahedral distortion. Solid circles indicate the occupied one-electron states, while the arrows indicate the spins.
TABLE II. Typical Hamiltonian parameters for $\mathrm{La}_{1-x} \mathrm{Ca}_{x} \mathrm{MnO}_{3}$ used in our calculation of exchange. Energies are in eV's. The onsite energy for oxygen $p$ orbitals $\epsilon_{p}$ is taken equal to zero, while the on-site energy for the $\mathrm{Mn}(d)$ orbitals depends on the charge state of the $\mathrm{Mn}$ atom (see Fig. 3). These parameters lead to a $\mathrm{MnO}_{6} p$-d charge transfer energy of $\Delta_{1}=\Delta_{2}=5 \mathrm{eV}$ for both $\mathrm{CaMnO}_{3}$ and $\mathrm{LaMnO}_{3}$ as discussed in the text. $V_{p d \pi}$ which appears in the $t_{2 g}$ hopping is taken to be $\approx-0.46 V_{p d \sigma}$ in accordance with Harrison's scaling.

\begin{tabular}{lllllllll}
\hline \hline$\epsilon_{p}$ & $\epsilon_{d}$ & $\epsilon_{d}^{\prime}$ & $\gamma$ & $\Delta_{J T}$ & $V_{p d \sigma}$ & $U_{p}$ & $U_{d}$ & $J_{H}$ \\
\hline 0 & 2 & -5 & 2 & 1.0 & 1.65 & 3.0 & 6.0 & 1 \\
\hline \hline
\end{tabular}

$\mathrm{Mn}^{3+}-\mathrm{O}-\mathrm{Mn}^{4+}$ case, are given by $\epsilon_{p}$ and $\epsilon_{d}$ for the $\mathrm{O}$ and the $\mathrm{Mn}$ atoms, respectively. Note that the on-site energies $\epsilon_{d}\left(\alpha, Q_{i}\right)$, where $\alpha$ goes over the five $d$ orbitals, depend on the charge state $Q\left(\mathrm{Mn}^{3+}\right.$ or $\left.\mathrm{Mn}^{4+}\right)$ of the $\mathrm{Mn}$ atom on account of the static Jahn-Teller distortion of the $\mathrm{MnO}_{6}$ octahedra, which is present at the $\mathrm{Mn}^{3+}$ site but not at $\mathrm{Mn}^{4+}$. Thus for the $\mathrm{Mn}^{3+}$ site, we have: $\epsilon_{d}\left(e_{g}^{1}, \mathrm{Mn}^{3+}\right)=\epsilon_{d}^{\prime}-\Delta_{J T}$ and $\epsilon_{d}\left(e_{g}^{2}, \mathrm{Mn}^{3+}\right)=\epsilon_{d}^{\prime}+\Delta_{J T}$, where $e_{g}^{1}$ and $e_{g}^{2}$ are the two Jahn-Teller-split $e_{g}$ orbitals, with $\Delta_{J T}$ being the Jahn-Teller one-electron energy gain at the $\mathrm{Mn}^{3+}$ site. For the $\mathrm{Mn}^{4+}$ site, by contrast, we will have $\epsilon_{d}\left(e_{g}^{1}, \mathrm{Mn}^{4+}\right)$ $=\epsilon_{d}\left(e_{g}^{2}, \mathrm{Mn}^{4+}\right)=\epsilon_{d}$, since there is no Jahn-Teller splitting there. Note that we have taken two different energies $\epsilon_{d}$ and $\epsilon_{d}^{\prime}$, for the $\mathrm{Mn}^{4+}$ and $\mathrm{Mn}^{3+}$, respectively, since quantities such as the local Madelung potential affecting on-site energies are in general site and crystal specific. The on-site energies for the $t_{2 g}$ orbitals are taken an amount $\gamma$ below the $e_{g}$ energies, which represents the crystal-field splitting. The magnitudes of the $\epsilon_{d}$ used in our calculations were inferred from the experimental charge transfer data ${ }^{17,18}$ and they have been listed in Table II together with other parameters.

Before we proceed to a discussion of the results, there is one more point that needs to be clarified in connection with the on-site energy $\epsilon_{d}\left(\alpha, Q_{i}\right)$. Consider, for example, the case of $\mathrm{Mn}^{4+}-\mathrm{O}-\mathrm{Mn}^{4+}$, which occurs in $\mathrm{CaMnO}_{3}$. Now, the ground state for this case will have perturbative contributions from a fermionic configuration, where a charge transfer has occurred from say the oxygen atom to a Mn atom, making it $\mathrm{Mn}^{3+}$. Now, if the $\mathrm{MnO}_{6}$ octahedron around this $\mathrm{Mn}^{3+}$ atom is allowed to respond to the electronic motion, the on-site $\operatorname{Mn}\left(e_{g}\right)$ energy for the added electron will be $\epsilon_{d}-\Delta_{J T}$, taking into account the Jahn-Teller energy gain $\Delta_{J T}$. Else, if the octahedron is assumed to be fixed in position, the energy will simply be $\epsilon_{d}$. We shall assume the latter, which is tantamount to assuming the electronic motion to be fast as compared to the lattice degrees of freedom. This is however not strictly true and the electron-lattice coupling does in fact have important consequences such as the oxygen-isotope effect. ${ }^{19}$ This type of electron-lattice coupling, the so-called dynamical Jahn-Teller effect, has been shown to reduce the magnitude of the exchange interaction and to lead to the oxygen-isotope effect. ${ }^{20,21}$

The typical parameter values for $\mathrm{La}_{1-x} \mathrm{Ca}_{x} \mathrm{MnO}_{3}$ that we shall use in this paper are given in Table II. The sign of the 
exchange [antiferromagnetic (AF) or ferromagnetic (FM)] is not sensitively dependent on the values of the parameters, so that the conclusions we derive from this work are quite robust. The magnitude of the exchange on the other hand does strongly depend on the parameters, e.g., it varies as the fourth power $t^{4}$ of the hopping parameter. In view of this, the magnitude of $t \equiv V_{p d \sigma}$ will be taken as a fitting parameter, by fitting the calculated value of $J$ to the experimental result for $\mathrm{CaMnO}_{3}$. The magnitude of $t$ needed for this is about 1.65 $\mathrm{eV}$, which is quite reasonable for the $V_{p d \sigma}$ hopping between manganese and oxygen.

To have a feel for the parameters, we compute the chargetransfer energies $\Delta_{1} \equiv \Delta\left(\mathrm{Mn}^{3+}-\mathrm{O}\right)$ and $\Delta_{2} \equiv \Delta\left(\mathrm{Mn}^{4+}-\mathrm{O}\right)$ from the total energy differences,

$$
\begin{gathered}
\Delta_{1}=E\left(d^{5} p^{5}\right)-E\left(d^{4} p^{6}\right)=\epsilon_{d}^{\prime}-\epsilon_{p}-5 U_{p}+\Delta_{J T}+4 U_{d}, \\
\Delta_{2}=E\left(d^{4} p^{5}\right)-E\left(d^{3} p^{6}\right)=\epsilon_{d}-\epsilon_{p}-5 U_{p}+3 U_{d} .
\end{gathered}
$$

In computing the charge-transfer energies above, the oxygen atoms are considered to be fixed as discussed earlier, i.e., the oxygen octahedra in the solid does not move with the fluctuation of the Mn valence. This is consistent with what might occur during experiments such as photoemission and optical conductivity measurements. From the parameters of Table II, we have $\Delta_{1}=\Delta_{2}=5 \mathrm{eV}$, which is consistent with the values deduced from photoemission ${ }^{17}$ and optical conductivity ${ }^{18}$ measurements. The experimental values are: $\Delta_{1} \sim 5 \mathrm{eV}, \Delta_{2}$ $\sim 2-3 \mathrm{eV}$, and $t_{p d} \sim 1.5 \mathrm{eV}$. Note that to obtain these chargetransfer energies, we need to invoke a material-dependent value for $\epsilon_{d}-\epsilon_{p}$ in Table II. In the solid such a term might originate naturally from such contributions as differences in the Madelung energy.

The ground-state energies $E_{\uparrow \uparrow}$ and $E_{\uparrow \downarrow}$ corresponding to the FM and AF alignments of the $\mathrm{Mn}\left(t_{2 g}\right)$ spins are obtained either by diagonalizing the Hamiltonian Eq. (1) exactly in the fermion-configuration space or by treating the electron hopping $t$ perturbatively using standard nondegenerate perturbation theory. If the $\mathcal{H}_{\mathrm{Hund}}=-J_{H} \Sigma_{i \alpha}^{\mathrm{Mn}} n_{i \alpha \uparrow} n_{i \alpha \downarrow}$ form, Eq. (4), for the Hund's energy is taken, then the FM and AF alignments are specified by the appropriate total number of the up and the down electrons $n_{\uparrow}$ and $n_{\downarrow}$ in the system. The exchange interaction $J$ between the Mn atoms is then obtained from the difference

$$
J=E_{\uparrow \uparrow}-E_{\uparrow \downarrow},
$$

where positive (negative) values of $J$ indicate an AF (FM) interaction between the $t_{2 g}$ spins. Note that our $J$ is related to $J^{\prime}$ via the equation $J=4 J^{\prime} S^{2}$, where $J^{\prime}$ appears in the standard expression for the Heisenberg Hamiltonian $H=$ $-2 J^{\prime} \Sigma_{i j} \vec{S}_{i} \cdot \vec{S}_{j}$.

For the perturbation theory results to reasonably converge we must have $t \ll \Delta E$, where $\Delta E$ is the separation of the excited state energies with respect to that of the ground state. This is not always true, so that we shall in general present the results of our exact calculations, although the perturbationtheory results will be used for general arguments.

In Fig. 4 we compare the results of the perturbation theory with the results of the exact diagonalization for the case of

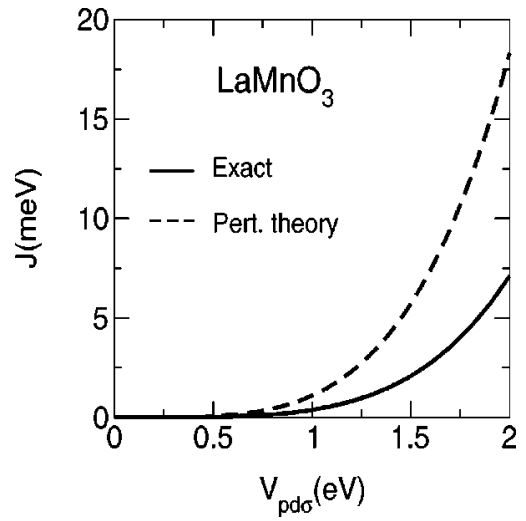

FIG. 4. Comparison of results of the perturbation theory to the results obtained from exact diagonalization for the case of $\mathrm{LaMnO}_{3}$ with $e_{g}^{\prime}-e_{g}^{\prime}$ orientations of the Mn orbitals. All parameters are the same as in Table II except that $J_{H}=\infty$.

$\mathrm{LaMnO}_{3}$ with $e_{g}^{\prime}-e_{g}^{\prime}$ orientations of the $\mathrm{Mn} e_{g}$ orbitals as an illustration. The perturbation theory reproduces the qualitative trend although the magnitude of the exchange interaction can be off by a factor of 2 or more depending on the parameters.

Throughout this paper, we have considered the magnetic exchange both with and without inclusion of the hopping to the $\operatorname{Mn}\left(t_{2 g}\right)$ orbitals. Analytical expressions for the fourthorder perturbation theory are, however, given for the case of no $t_{2 g}$ hopping, since otherwise the expressions become quite tedious and lengthy. The full results of the perturbation theory including the effects of the $t_{2 g}$ hopping have been calculated numerically, whenever needed. Exact solution for the ground-state energies have been obtained by either the standard diagonalization method or by the Lanczos method if the size of the Hamiltonian matrix is too large.

\section{EXCHANGE INTERACTION IN La ${ }_{1-x} \mathrm{Ca}_{x} \mathrm{MnO}_{3}$}

As shown in Fig. 1, manganites exhibit a variety of magnetic structures. ${ }^{10-12} \mathrm{LaMnO}_{3}$ is a type $A$ antiferromagnet, where the $\operatorname{Mn}\left(t_{2 g}\right)$ core spins are arranged ferromagnetically within the $a b$ planes and are aligned antiparallel between adjacent $a b$ planes. By contrast, $\mathrm{CaMnO}_{3}$ is a type $G$ antiferromagnet, where all nearest-neighbor spins are aligned antiferromagnetically. The intermediate compound $\mathrm{La}_{1 / 2} \mathrm{Ca}_{1 / 2} \mathrm{MnO}_{3}$ is ordered according to the $\mathrm{CE}$ structure, which is charge ordered and has ferromagnetic chains that zigzag in the $a b$ plane, with identical $a b$ planes stacked along the $c$ direction except that the spins are reversed from one plane to another.

The Goodenough-Kanamori-Anderson (GKA) Rules. In the late 1950s Anderson, ${ }^{3}$ Goodenough, ${ }^{1,4}$ and Kanamori ${ }^{2}$ developed a set of semiempirical rules that give the sign and the relative magnitude of the exchange interaction mediated by an intermediate ion. In the case of a straight $\mathrm{Mn}-\mathrm{O}-\mathrm{Mn}$ bond, these rules may be stated.

(i) When the $e_{g}$ orbitals of the two cations are both partially filled with one electron each and the $e_{g}$ orbitals point towards each other such that we have a large Mn-O $p$ - $d$ 
overlap, the exchange is antiferromagnetic and comparatively large.

(ii) When the two cations have empty $e_{g}$ orbitals the net exchange, mediated via the $t_{2 g}$ orbitals, is also antiferromagnetic, although its magnitude is relatively small.

(iii) When one cation has empty $e_{g}$ orbitals while the other has one $e_{g}$ electron, there are two scenarios: (a) If the occupied $e_{g}$ orbital points towards the oxygen, so that there is a large overlap, the net exchange is ferromagnetic and moderately strong, and (b) if the occupied $e_{g}$ is oriented such that its overlap with the oxygen is negligible, then we have a situation rather similar to case (ii), which leads to an antiferromagnetic exchange.

However, the GKA rules are too general and caution has to be taken to apply them to specific cases, since the sign of the exchange is controlled not just by the occupation of the orbitals but also by the relative strengths of the various electronic parameters in the system. The cancellations of the various ferromagnetic and antiferromagnetic pieces in the exchange interaction have in fact been implicitly included in formulating the above rules.

\section{A. $\mathrm{CaMnO}_{3}$}

\section{Exchange interaction with $J_{H}=\infty$ and no $t_{2 g}$ hopping}

We first consider the case of $\mathrm{CaMnO}_{3}$ with $J_{H}=\infty$ and no $t_{2 g}$ hopping. These approximations are often made for the manganites making this case worthwhile to consider. However, as will be shown shortly, the magnitude of the exchange is substantially affected by these approximations.

In $\mathrm{CaMnO}_{3}$ the Mn-O-Mn triad has the nominal valence of $\mathrm{Mn}^{4+}-\mathrm{O}^{2-}-\mathrm{Mn}^{4+}$. With $t_{2 g}$ hopping neglected, these electrons can be treated as core, and furthermore since $J_{H}$ $=\infty$, the $e_{g}$ orbitals with spin antiparallel to the core $t_{2 g}$ spins are not occupied. Now, retaining the $\mathrm{O}(p)$ and $\operatorname{Mn}(d)$ orbitals in enumerating the many-electron configurations accessible to the system, the total number of configurations for

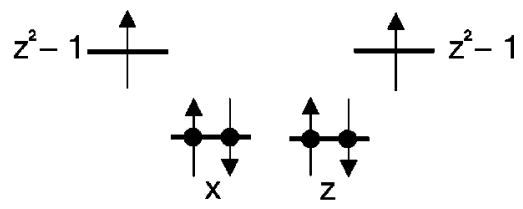

(a)

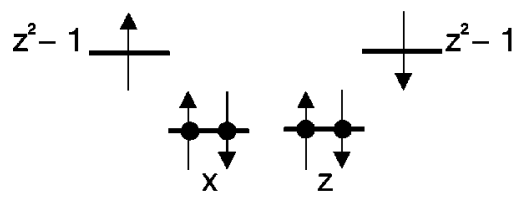

(b)

FIG. 5. Orbitals considered in forming the Hamiltonians $H_{\uparrow \uparrow}$ and $H_{\downarrow \downarrow}$ of Eqs. (8) and (9) appropriate for $\mathrm{CaMnO}_{3}$. (a) corresponds to the FM alignment of the two Mn core spins while (b) corresponds to the $A F$ alignment. Solid circles indicate states occupied by an electron.

the FM alignment of the $\mathrm{Mn}$ atoms is given by ${ }^{n} C_{N} \times{ }^{m} C_{M}$ $={ }^{7} C_{3} \times{ }^{3} C_{3}=35$ ( $n$ spin-up electrons distributed among $N$ available spin-up orbitals and $m$ spin-down electrons distributed among $M$ spin-down orbitals). This results in a $35 \times 35$ Hamiltonian matrix, the size of which can be further reduced if the coordinate system is properly chosen.

Choosing local coordinate systems on the two Mn atoms such that the $z$ axis points towards the oxygen atom, one finds that both the $\mathrm{O}\left(p_{y}\right)$ and the $\mathrm{Mn}\left(x^{2}-y^{2}\right)$ orbitals do not couple to the rest of the system. This makes the total number of configurations of a manageable size, viz., ${ }^{4} C_{2} \times{ }^{2} C_{2}=6$ (see Fig. 5). Taking these configurations in the following order: $|1100\rangle,|1010\rangle,|1001\rangle,|0110\rangle,|0101\rangle$, and $|0011\rangle$, where the four numbers in the ket indicate the occupation numbers for the oxygen $p_{x}$ and $p_{z}$ orbitals, and the $e_{g}$ orbitals on the left and the right $\mathrm{Mn}$ atoms, in that order, the $6 \times 6$ Hamiltonian matrix is given by

$$
H_{\uparrow \uparrow}=\left(\begin{array}{cccccc}
6 U_{p} & t_{3} & t_{4} & -t_{1} & -t_{2} & 0 \\
t_{3} & \epsilon_{d}+3 U_{p} & 0 & 0 & 0 & -t_{2} \\
t_{4} & 0 & \epsilon_{d}+3 U_{p} & 0 & 0 & t_{1} \\
-t_{1} & 0 & 0 & \epsilon_{d}+3 U_{p} & 0 & -t_{4} \\
-t_{2} & 0 & 0 & 0 & \epsilon_{d}+3 U_{p} & t_{3} \\
0 & -t_{2} & t_{1} & -t_{4} & t_{3} & 2 \epsilon_{d}+U_{p}
\end{array}\right) .
$$

Here, $t_{1}=\left\langle x \mid e_{g}(l)\right\rangle, t_{2}=\left\langle x \mid e_{g}(r)\right\rangle, t_{3}=\left\langle z \mid e_{g}(l)\right\rangle$, and $t_{4}=\left\langle z \mid e_{g}(r)\right\rangle$, where $l(r)$ denotes the left (right) Mn atom. Note that the above Hamiltonian is the same irrespective of which of the two forms of $\mathcal{H}_{\text {Hund }}$ is used, since $J_{H}=\infty$.

The corresponding Hamiltonian matrix for the AF case is given by 


$$
H_{\uparrow \downarrow}=\left(\begin{array}{ccccccccc}
6 U_{p} & t_{4} & -t_{2} & t_{3} & 0 & 0 & -t_{1} & 0 & 0 \\
t_{4} & \epsilon_{d}+3 U_{p} & 0 & 0 & t_{3} & 0 & 0 & -t_{1} & 0 \\
-t_{2} & 0 & \epsilon_{d}+3 U_{p} & 0 & 0 & t_{3} & 0 & 0 & -t_{1} \\
t_{3} & 0 & 0 & \epsilon_{d}+3 U_{p} & t_{4} & -t_{2} & 0 & 0 & 0 \\
0 & t_{3} & 0 & t_{4} & 2 \epsilon_{d}+U_{p} & 0 & 0 & 0 & 0 \\
0 & 0 & t_{3} & -t_{2} & 0 & 2 \epsilon_{d}+U_{p} & 0 & 0 & 0 \\
-t_{1} & 0 & 0 & 0 & 0 & 0 & \epsilon_{d}+3 U_{p} & t_{4} & -t_{2} \\
0 & -t_{1} & 0 & 0 & 0 & 0 & t_{4} & 2 \epsilon_{d}+U_{p} & 0 \\
0 & 0 & -t_{1} & 0 & 0 & 0 & -t_{2} & 0 & 2 \epsilon_{d}+U_{p}
\end{array}\right) .
$$

The difference in the lowest eigenvalues to the fourth order in the perturbation theory is found to be

$$
J^{33}=E_{\uparrow \uparrow}-E_{\uparrow \downarrow}=\frac{4 t^{4} \cos ^{2} \theta}{\Delta_{2}^{2}\left(2 \Delta_{2}+U_{p}\right)} .
$$

Here and throughout the paper, the superscripts in $J^{\mu \nu}$ indicate the number of $d$ electrons on the two Mn atoms. Thus $J^{33}$ is appropriate for $\mathrm{CaMnO}_{3}$ and $J^{44}$ for $\mathrm{LaMnO}_{3}$, and so on.

It is interesting to note from Eq. (10) that the exchange interaction has always a positive sign, since the charge transfer and the Coulomb energies, $\Delta$ and $U_{p}$, are both positive, leading to an antiferromagnetic exchange, irrespective of the magnitudes of the parameters. A similar AF interaction has been obtained earlier by Millis for a straight Mn-O-Mn bond and ignoring the degeneracy of the $\mathrm{O}(p)$ orbitals. ${ }^{22}$ The results of the perturbation theory are compared to those obtained from diagonalization in Fig. 6, where the exchange interaction is shown as a function of the hopping integral. The exact results deviate from the perturbation-theory results for large hopping as expected.

\section{Exchange with finite $J_{H}$}

Now we relax the condition that $J_{H}=\infty$. The strength of the exchange is expected to diminish as $J_{H}$ is reduced from

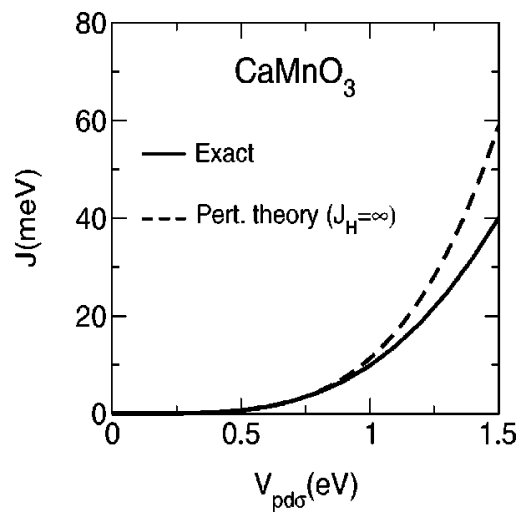

FIG. 6. Exchange interaction as a function of the $p-d$ hopping for $\mathrm{CaMnO}_{3}$ with $t_{2 g}$ hopping neglected: (a) Perturbation theory (dashed line) and (b) exact results (solid line). infinity. In fact, it is strictly zero in the limit $J_{H} \rightarrow 0$, if the hopping of the $t_{2 g}$ electrons is not allowed. This is because in that case the $t_{2 g}$ electrons can be treated as core spins that are decoupled from the rest of the system, $J_{H}$ being 0 , and it then does not matter what the relative orientations of the $t_{2 g}$ spins are. This can be most clearly seen from the $\vec{S} \cdot \vec{\sigma}$ form of the Hund's enegy Eq. (5).

The magnitude of the Hund's-rule energy in manganites is $J_{H} \approx 1 \mathrm{eV}^{7}$ To illustrate the dependence of $J_{H}$ on the exchange, we consider the case of $\mathrm{CaMnO}_{3}$.

For simplicity, we first neglect the $t_{2 g}$ hopping, take the $\vec{S} \cdot \vec{\sigma}$ form of the Hund's enegy Eq. (5), and consider the case of the straight Mn-O-Mn bond $\theta=180^{\circ}$. It then turns out that we need only consider explicitly the oxygen $z$ and the manganese $z^{2}-1$ orbitals (six orbitals in total including spin) in the many-particle configuration, which leads to a $9 \times 9$ Hamiltonian matrix for both AF and FM cases. This can be diagonalized numerically and the results are shown in Fig. 7.

We have also obtained an expression for the exchange using the fourth-order perturbation theory, so that the functional dependence of $J_{H}$ can be explicitly seen,

$$
\begin{aligned}
J^{33}\left(J_{H}\right)= & \frac{4 t^{4}}{\Delta_{2}^{2}\left(2 \Delta_{2}+U_{p}\right)}+\frac{4 t^{4}}{\Delta_{2}^{\prime 2}\left(2 \Delta_{2}^{\prime}+U_{p}\right)} \\
& -\frac{2 t^{4}}{\left(2 \Delta_{2}+U_{p}+3 J_{H}\right)} \times\left(\frac{1}{\Delta_{2}}+\frac{1}{\Delta_{2}^{\prime}}\right)^{2} .
\end{aligned}
$$

Here $\Delta_{2}$ is the $p$ - $d$ charge transfer energy defined in Eq. (6) and $\Delta_{2}^{\prime}=\Delta_{2}+3 J_{H}$. Notice that our earlier result Eq. (10) is consistent with Eq. (11) in the limit of $J_{H}=\infty$ and $\theta=180^{\circ}$.

As expected, the perturbation results show a diminishing $J$ as $J_{H}$ is decreased from infinity. This behavior is also reproduced from the exact results, shown in Fig. 7 for both forms of the Hund's energy. By contrast, if the $t_{2 g}$ hopping is allowed, there will be a difference between the ground-state energies for the FM and AF configurations even when $J_{H}$ $=0$, since the $t_{2 g}$ spins are still coupled to the rest of the system via electron hopping. $J$ thus does not go to zero for $J_{H}=0$, but takes a nonzero value as seen from Fig. 7 . 


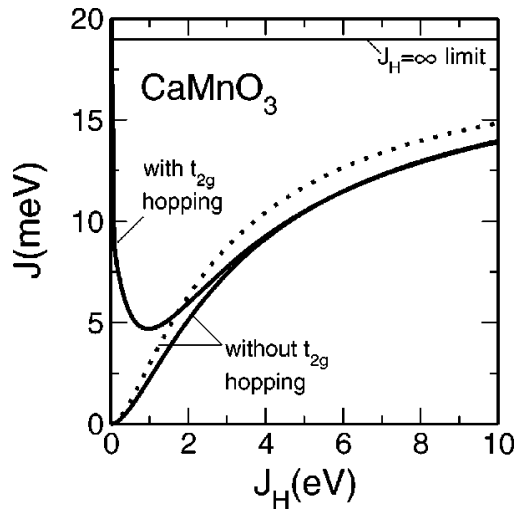

FIG. 7. Effect of a finite Hund's coupling $J_{H}$ on the exchange interaction $J$ as obtained from exact diagonalization. Both forms of $\mathcal{H}_{\text {Hund }}$ are considered: (1) $\mathcal{H}_{\text {Hund }}=-J_{H} \Sigma_{i}^{\mathrm{Mn}} \vec{S}_{i} \cdot \vec{\sigma}_{i}$ (dashed line) and (2) $\mathcal{H}_{\text {Hund }}=-J_{H} \sum_{i \alpha}^{\mathrm{Mn}} n_{i \alpha \uparrow} n_{i \alpha \downarrow}$ (solid lines). The latter case was studied both with and without the $t_{2 g}$ hopping. Parameters are the same as in Table II except that here $V_{p d \sigma}=1.2 \mathrm{eV}$. The figure shows three main results. (i) Both forms of the Hund's energy produce similar results when there is no $t_{2 g}$ hopping. (ii) A finite $J_{H}(\sim 1 \mathrm{eV}$ in typical solids) significantly reduces the magnitude of $J$ as compared to the $J_{H} \rightarrow \infty$ value. (iii) The $t_{2 g}$ hopping substantially affects the magnitude of exchange, if $J_{H}$ is less than several eV's. Note that only the second form of the Hund's energy can be used when $t_{2 g}$ hopping is present. All three curves go to the same limit as $J_{H}$ $\rightarrow \infty$ as indicated in the figure.

\section{Bond-angle dependence of exchange}

Figure 8 shows the bond-angle dependence of the exchange for $\mathrm{CaMnO}_{3}$ and also the effect of a finite value of $J_{H}$ as well as the effect of the $t_{2 g}$ hopping on the exchange. The magnitude of the exchange decreases as the angle is decreased from $180^{\circ}$, roughly going as $\cos ^{2} \theta$ as suggested by the perturbation theory results Eq. (10).

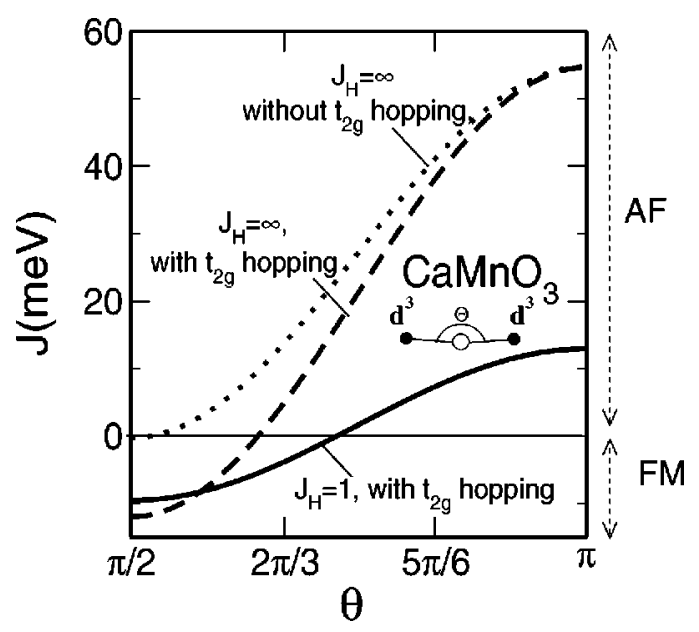

FIG. 8. Variation of the exchange interaction in $\mathrm{CaMnO}_{3}$ with the bond angle $\theta$. Shown are the results for three different cases: (a) $J_{H}=\infty$ and without $t_{2 g}$ hopping (dotted line), (b) $J_{H}=\infty$ and with $t_{2 g}$ hopping (dashed line), and (c) $J_{H}=1 \mathrm{eV}$ and with $t_{2 g}$ hopping (full line). Parameters other than $J_{H}$ are the same as in Table II. Notice the switching of the sign of the exchange around $132^{\circ}$ for parameters appropriate for $\mathrm{CaMnO}_{3}$.
If the $t_{2 g}$ hopping is neglected, then the antiferromagnetic interaction between the $t_{2 g}$ spins in $\mathrm{CaMnO}_{3}$ is caused by the subtle differences of the hopping of the $\mathrm{O}(p)$ electrons to the empty $e_{g}$ levels. For the FM case, only three of the six $\mathrm{O}(p)$ electrons can hop to the $\mathrm{Mn}$ atoms, while in the AF case, all six electrons can participate in the hopping process. The latter case where all six electrons can hop turns out to be energetically favorable leading to an AF coupling as given by Eq. (10).

To this the $t_{2 g}$ hopping adds two competing interactions: (i) The $t_{2 g}-e_{g}$ hopping is ferromagnetic, because this hopping is more effective in lowering the ground-state energy, if the $e_{g}$ orbital on the second $\mathrm{Mn}$ atom has the same spin, which in turn requires that the two $t_{2 g}$ spins be ferromagnetically aligned, and (ii) the $t_{2 g}-t_{2 g}$ hopping between the occupied levels of one Mn atom and the unoccupied levels of the other is antiferromagnetic by a similar argument. In fact, if $J_{H}=\infty$, there is no $t_{2 g}-t_{2 g}$ hopping and the former of the two competing interactions remains. This effect is seen in Fig. 8 and is in fact precisely the difference between the dotted curve and the dashed curve. The full line in that figure has all interactions and also there, $J_{H}$ is taken to be $1 \mathrm{eV}$ as appropriate for the manganites.

Notice also that in Fig. 8 there is a crossover between AF and FM as a function of the Mn-O-Mn bond angle $\theta$, which is about $132^{\circ}$ for the parameters used. The possibility of such a crossover is long known since the early work of Goodenough $^{12}$ and recently such a crossover has been experimentally observed in a somewhat different system $\mathrm{SeCuO}_{3}{ }^{23}$

The experimental $J^{\mathrm{CaMnO}_{3}} \approx 13.1 \mathrm{meV}$, estimated from the Neel temperature $T_{N} \approx 110 \mathrm{~K},{ }^{10,24}$ agrees with the results of Fig. 6 if we take $t \equiv V_{p d \sigma} \approx 1.65 \mathrm{eV}$. This value of $t$ is used throughout the paper. As already mentioned, the magnitude of $J$ is sensitively dependent on $t$, while the sign of the interaction is always antiferromagnetic consistent with the observed type $G$ structure in $\mathrm{CaMnO}_{3}$.

\section{B. $\mathrm{LaMnO}_{3}$}

Now, we turn to the case of $\mathrm{LaMnO}_{3}$, which is, unlike $\mathrm{CaMnO}_{3}$, somewhat more complicated because of the three different possible orientations of the occupied $e_{g}$ orbitals, depending on the specific Jahn-Teller distortion at the $\mathrm{Mn}^{3+}$ sites. Here, we have the nominal valence $\mathrm{Mn}^{3+}-\mathrm{O}^{2-}-\mathrm{Mn}^{3+}$, with $\operatorname{Mn}\left(d^{4}\right)$ configurations, which can be either $t_{2 g}^{3} e_{g}^{1}$ if the $\mathrm{Mn}$ atom is on a "long" Mn-O bond or $t_{2 g}^{3} e_{g}^{\prime 1}$ if the Mn-O bond is "short" (see Fig. 2). The orientation of the $e_{g}$ orbitals is dictated by the specific Jahn-Teller distortion of the surrounding $\mathrm{MnO}_{6}$ octahedra and this in turn determines the magnetic coupling.

\section{Exchange interaction with $J_{H}=\infty$ and no $t_{2 g}$ hopping}

We first discuss the case, where $J_{H}=\infty$ and the $t_{2 g}$ hopping is suppressed. This is important to consider because analytical expressions for the exchange can be obtained from perturbation theory in this approximation and they capture the essence of the results obtained from exact diagonalization of the full Hamiltonian like in the case of $\mathrm{CaMnO}_{3}$. 


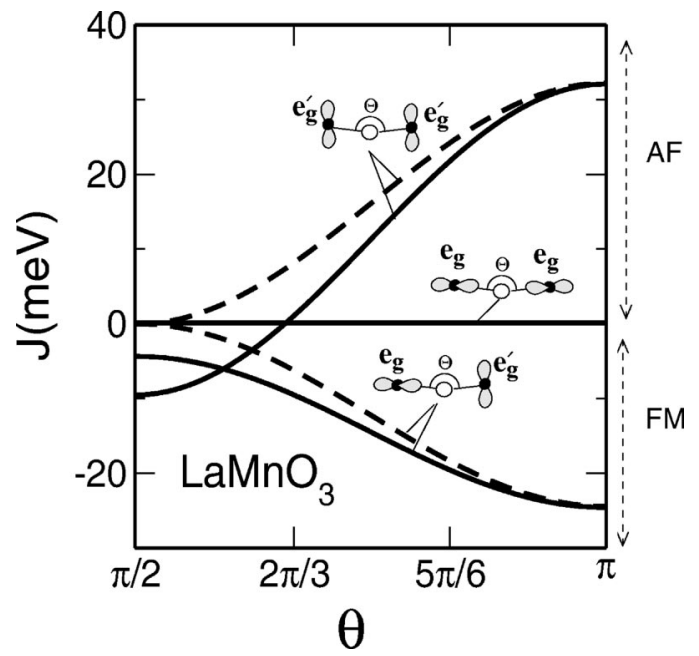

FIG. 9. Calculated exchange interaction in $\mathrm{LaMnO}_{3}$ with $J_{H}$ $=\infty$. The magnetic exchange is zero for the $\operatorname{Mn}\left(e_{g}-e_{g}\right)$ configuration in our model. The exchange interaction for the $\mathrm{Mn}\left(e_{g}^{\prime}-e_{g}^{\prime}\right)$ case is antiferromagnetic, which corresponds to the case of Mn atoms located on the neighboring planes in $\mathrm{LaMnO}_{3}$ along the $c$ axis. The Mn $\left(e_{g}-e_{g}^{\prime}\right)$ coupling is, by contrast, ferromagnetic as observed for the case of the $\mathrm{Mn}$ atoms in the $a b$ plane. The inclusion of the $t_{2 g}$ hopping adds a ferromagnetic component to the exchange interaction as discussed in the text. In the case of the $e_{g}^{\prime}-e_{g}^{\prime}$ orientation, the $t_{2 g}$ hopping leads to an exchange interaction which changes from ferromagnetic to antiferromagnetic as the angle $\theta$ is varied. Solid (dashed) lines are with (without) $t_{2 g}$ hopping included.

When the core $t_{2 g}$ spins in the Mn atoms are ferromagnetically aligned, there are six spin-up orbitals [the two $e_{g}$ orbitals on each of the Mn atoms and $\mathrm{O}\left(p_{x}\right)$ and $\left.\mathrm{O}\left(p_{z}\right)\right]$ and two spin-down orbitals $\left[\mathrm{O}\left(p_{x}\right)\right.$ and $\left.\mathrm{O}\left(p_{z}\right)\right]$ to be occupied by four spin-up electrons and two spin-down electrons, leading to a fifteen-dimensional $\left({ }^{6} C_{4} \times{ }^{2} C_{2}\right)$ configuration space. Notice that neither the $t_{2 g}$ nor the $\mathrm{O}\left(p_{y}\right)$ electrons are counted since they are effectively uncoupled to the rest of the system. Similarly, for the antiferromagnetic alignment of the $\mathrm{Mn}$ core spins, there are four spin-up and an equal number of spin-down orbitals to be occupied by three spin-up and the same number of spin-down electrons, leading to a sixteendimensional $\left({ }^{4} C_{3} \times{ }^{4} C_{3}\right)$ configuration space. The exact results are obtained by simply diagonalizing these matrices and taking the difference in the ground-state energies as per Eq. (7). The exchange energy thus obtained is plotted in Fig. 9 for the three different cases of the relative orientations of the $\operatorname{Mn}\left(e_{g}\right)$ orbitals.

The expression for the exchange energy obtained from perturbation theory applicable to the $\operatorname{Mn}\left(d^{4}\right)-\mathrm{O}-\mathrm{Mn}\left(d^{4}\right)$ situation in $\mathrm{LaMnO}_{3}$ is given by

$$
J^{44}=\frac{1}{\left(\Delta_{1}+\Delta_{J T}\right)^{2}}\left(\frac{-T_{1}^{2}}{U_{d}+\Delta_{J T}}+\frac{4 T_{2}^{2}}{2\left(\Delta_{1}+\Delta_{J T}\right)+U_{p}}\right),
$$

where

$$
\begin{gathered}
T_{1}=\sum_{p=x, z} t_{p, e_{l}} t_{p, E_{r}}+t_{p, e_{r}} t_{p, E_{l}}, \\
T_{2}=\sum_{p=x, z} t_{p, E_{r}} t_{p, E_{l}} .
\end{gathered}
$$

Here $t_{\alpha \beta}$ are the hopping parameters $\langle\alpha|H| \beta\rangle, e_{l}\left(e_{r}\right)$ denotes the occupied $e_{g}$ orbital on the left (right) Mn atom, and $E_{l}\left(E_{r}\right)$ denotes the corresponding empty $e_{g}$ orbital. The $e_{l}$ and $e_{r}$ orbitals can have one of the three possible, $e_{g}, e_{g}^{\prime}$, or $e_{g}^{\prime \prime}$, orientation as discussed before. $T_{1}$ and $T_{2}$, which may be evaluated using Table I, contain the dependence on the Mn$\mathrm{O}-\mathrm{Mn}$ angle $\theta$ and on the orbital orientations.

Equation (12) shows the competition between ferromagnetic first term and antiferromagnetic second term contributions to the exchange. As seen from Eqs. (13) and (14), the ferromagnetic term involves both the occupied $\operatorname{Mn}\left(d_{z^{2}-1}\right)$ as well as the unoccupied $\operatorname{Mn}\left(d_{x^{2}-y^{2}}\right)$ orbitals, denoted by $e$ and $E$ respectively, while the antiferromagnetic term involves only the empty $\operatorname{Mn}\left(d_{x^{2}-y^{2}}\right)$ orbitals. The antiferromagnetic interaction thus originates from the virtual hopping of the $\mathrm{O}(p)$ electrons to the unoccupied $\mathrm{Mn}(d)$ levels. Now, if the two Mn core spins are antiferromagnetically aligned, then $\mathrm{O}(p)$ electrons of both spins can hop to a Mn atom, while for the FM alignment, only one type of spin can participate in the hopping process. The energy in the two cases differs only in the fourth order in the perturbation theory, resulting in the second term in Eq. (12). The ferromagnetic term there is consistent with the double-exchange idea of Anderson and Hasegawa ${ }^{25}$ and of de Gennes, ${ }^{26}$ where $T_{1}$ can be thought of an effective Mn-Mn hopping, while the expression for $T_{2}$ is indicative of the origin of double exchange from simultaneous double hops as originally envisaged by Zener. ${ }^{27}$

As Eq. (12) shows, the net exchange in the $d^{4}-d^{4}$ system can either be ferromagnetic or antiferromagnetic depending on which of the two terms dominates. Indeed in the observed type- $A$ structure for $\mathrm{LaMnO}_{3}$, the exchange is sometimes ferromagnetic (intraplane) and sometimes antiferromagnetic (interplane). The net exchange may be obtained by computing the magnitudes of the hoppings $T_{1}$ and $T_{2}$ using the parameters in Table I for the different $J T$-induced orientations of the $\operatorname{Mn}\left(e_{g}\right)$ orbitals, viz., $e_{g}, e_{g}^{\prime}$, or $e_{g}^{\prime \prime}$.

Filling in the hopping parameters from Table I, we find that the Mn-O-Mn bond with $e_{g}-e_{g}$ configuration has zero exchange,

$$
J^{44}\left(e_{g}-e_{g}\right)=0,
$$

for all bond angles. This is easily understood by noting that the $e_{g}-e_{g}$ configuration allows only electronic hopping from the oxygen to the $e_{g}$ orbitals (i. e., the $z^{2}-1$ orbital pointing along $\mathrm{Mn}-\mathrm{O}$ bond), which are already occupied in the present case. The hopping to the corresponding $x^{2}-y^{2}$ orbitals to the $\mathrm{O}(p)$ orbitals is zero by symmetry, which may be easily seen by considering a new set of $\mathrm{O}(p)$ basis states, viz., a $p$ orbital directed along the $\mathrm{Mn}-\mathrm{O}$ bond, one perpendicular to the plane of the triad, and the third $p$ orbital orthogonal to both these. This is true both for the AF case and the FM case and also whether the $t_{2 g}$ hopping is present or 
not. Consequently, the exchange interaction is zero. This particular type of orbital configuration is however not encountered in $\mathrm{LaMnO}_{3}$.

The configurations encountered in $\mathrm{LaMnO}_{3}$ are the $e_{g}-e_{g}^{\prime}$ configuration, as realized in the (001) planes, and the $e_{g}^{\prime}-e_{g}^{\prime}$ configuration encountered for two $\mathrm{Mn}$ atoms on neighboring planes. For the former case, one finds $T_{1}=(\sqrt{3} / 2)$ $\times t^{2} \cos (\theta)$ and $T_{2}=0$, so that Eq. (12) indicates that the exchange is always ferromagnetic,

$$
J^{44}\left(e_{g}-e_{g}^{\prime}\right)=-\frac{3 t^{4} \cos ^{2} \theta}{4\left(\Delta_{1}+\Delta_{J T}\right)^{2}\left(U_{d}+\Delta_{J T}\right)} .
$$

The lack of an antiferromagnetic contribution is again due to the suppression of the virtual hopping of the $\mathrm{O}(p)$ electron to the unoccupied $\mathrm{Mn}$ orbitals because of symmetry and the fact that $J_{H}$ is large tending to $\infty$. Note that the sign of the ferromagnetic exchange indicated by Eq. (16) is independent of both the electronic parameters as well as the Mn-O-Mn bond angle within our model. Thus the model explains the FM exchange coupling in the $a b$ planes of $\mathrm{LaMnO}_{3}$ and this can be thought of as the Anderson-Hasegawa double exchange arising from the motion of the $\operatorname{Mn}(d)$ electrons to the empty $d$ levels. Our model would therefore predict a universal ferromagnetic in-plane coupling provided that the $\operatorname{Mn}\left(d^{4}\right)$ atoms occur in the $e_{g}-e_{g}^{\prime}$ configuration.

Next, we turn to the $e_{g}^{\prime}-e_{g}^{\prime}$ configuration that occurs along the [001] direction in the case of $\mathrm{LaMnO}_{3}$, i.e., where the lobes of neither of the two occupied " $z^{2}-1$ " orbitals point towards the oxygen. In this case, we find that there is both a FM and an AF contribution, $T_{1}=(2 / \sqrt{3}) T_{2}=(\sqrt{3} / 2) t^{2} \cos \theta$, so that Eq. (12) leads to

$$
\begin{aligned}
J^{44}\left(e_{g}^{\prime}-e_{g}^{\prime}\right)= & \frac{3 t^{4} \cos ^{2} \theta}{4\left(\Delta_{1}+\Delta_{J T}\right)^{2}} \\
& \times\left[-\frac{1}{U_{d}+\Delta_{J T}}+\frac{3}{2\left(\Delta_{1}+\Delta_{J T}\right)+U_{p}}\right] .
\end{aligned}
$$

The net exchange could be either FM or AF. However, for any reasonable choice of the parameters for the manganites, the exchange turns out to be antiferromagnetic. This is consistent with the interplane AF interaction in $\mathrm{LaMnO}_{3}$.

The exchanges described by Eqs. (16) and (17) are relevant to $\mathrm{LaMnO}_{3}$, where they successfully describe the inplane ferromagnetic and the interplane antiferromagnetic coupling. The situation with the $e_{g}^{\prime \prime}$ configuration of the Mn atom does not arise in the $\mathrm{LaMnO}_{3}$. However, they may be relevant in other compounds. Within the fourth-order perturbation theory, we find these to be

$$
J^{44}\left(e_{g}-e_{g}^{\prime \prime}\right)=J^{44}\left(e_{g}-e_{g}^{\prime}\right)
$$

and

$$
J^{44}\left(e_{g}^{\prime}-e_{g}^{\prime \prime}\right)=J^{44}\left(e_{g}^{\prime \prime}-e_{g}^{\prime \prime}\right)=J^{44}\left(e_{g}^{\prime}-e_{g}^{\prime}\right)
$$

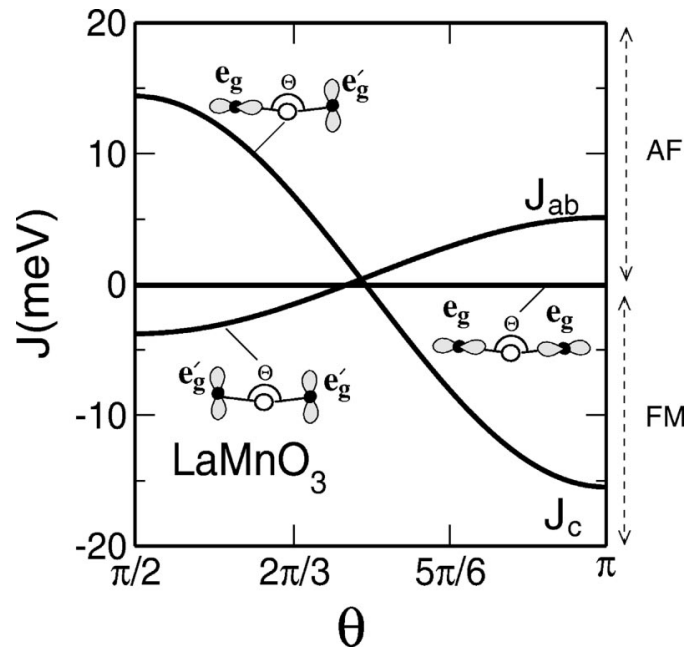

FIG. 10. Exchange interaction in $\mathrm{LaMnO}_{3}$ with the full Hamiltonian, i.e., with $J_{H}=1 \mathrm{eV}$ and with $t_{2 g}$ hopping included. The intraplane and the interplane exchanges, $J_{a b}$ and $J_{c}$, correspond to the $e_{g}-e_{g}^{\prime}$ and $e_{g}^{\prime}-e_{g}^{\prime}$ orbital orientations, respectively.

where the expressions for $J^{44}\left(e_{g}-e_{g}^{\prime}\right)$ and $J^{44}\left(e_{g}^{\prime}-e_{g}^{\prime}\right)$ are given by Eqs. (16) and (17).

In Fig. 9, we show the exchange interaction for $\mathrm{LaMnO}_{3}$ with $J_{H}=\infty$ calculated from the exact diagonalization, both with and without the $t_{2 g}$ hopping. The perturbative results presented above were obtained for the latter case, i.e., with neglect of the $t_{2 g}$ hopping. When this coupling is taken into consideration, it adds an extra ferromagnetic component as seen from the figure. The calculated exchanges for $\mathrm{LaMnO}_{3}$ obtained from exact diagonalization are shown in Fig. 9 and they follow the general trend obtained from the results of the perturbation theory.

\section{Exchange interaction with the full Hamiltonian}

The exchange interactions for the $\mathrm{Mn}^{3+}-\mathrm{O}-\mathrm{Mn}^{3+}$ case appropriate for $\mathrm{LaMnO}_{3}$ are shown in Fig. 10 as calculated from the exact diagonalization of the full Hamiltonian, i.e., with $J_{H}=1 \mathrm{eV}$ and with all hoppings including the $t_{2 g}$ hopping present. For the straight Mn-O-Mn bond $\left(\theta=180^{\circ}\right)$, the intraplane exchange $J_{a b}$ is ferromagnetic and the interplane exchange $J_{c}$ is antiferromagnetic exactly as observed in $\mathrm{LaMnO}_{3}$. Notice also from the figure that the sign of the exchange is reversed if the Mn-O-Mn bond is bent beyond the value of $\theta \leqslant 135^{\circ}$ or so. The calculated magnitudes of the $J$ 's are compared to the experimental as well as earlier theoretical results in Table III for the compound $\mathrm{LaMnO}_{3}$. The signs of the $J$ 's agree with the experimental results as well as with the theoretical results of Su et al. ${ }^{6}$ obtained from the Hartree-Fock calculations. The magnitudes of the $J$ 's are satisfactory compared to the experimental values, given the simplicity of the model and considering the fact that the magnitudes are very sensitive to the strength of the electron hopping.

\section{C. $\mathrm{La}_{1 / 2} \mathrm{Ca}_{1 / 2} \mathrm{MnO}_{3}$}

The compound $\mathrm{La}_{1 / 2} \mathrm{Ca}_{1 / 2} \mathrm{MnO}_{3}$ has the peculiar type-CE structure as shown in Fig. 1(c) and here too we find that all exchange interactions are correctly described within our model. 
TABLE III. Comparison of the exchange interaction energies $J$ 's for $\mathrm{LaMnO}_{3}$ obtained from the present theory with those obtained from Hartree-Fock [Ref. 6] and Density-Functional [Ref. 5] calculations and from inelastic neutron scattering experiments [Ref. 28] in $\mathrm{meV}$.

\begin{tabular}{lcccc}
\hline \hline Exp. & Present theory & $\begin{array}{c}\text { Hartree-Fock } \\
\text { approximation }\end{array}$ & $\begin{array}{c}\text { Local spin density } \\
\text { approximation }\end{array}$ \\
\hline$J_{a b}$ & -13.4 & -15.5 & -7.0 & -18.2 \\
$J_{c}$ & 9.7 & 5.1 & 1.7 & -6.2 \\
\hline \hline
\end{tabular}

First we consider the interplanar exchange between two $\mathrm{Mn}$ atoms on neighboring $a b$ planes in the $c$ direction. There are two types of interplanar coupling, viz., (i) $\mathrm{Mn}^{4+}-\mathrm{O}^{2-}-\mathrm{Mn}^{4+}$ just like in $\mathrm{CaMnO}_{3}$ and (ii) $\mathrm{Mn}^{3+}-\mathrm{O}^{2-}-\mathrm{Mn}^{3+}$ with the $e_{g}^{\prime}-e_{g}^{\prime}$ orbital orientation like in the case of $\mathrm{LaMnO}_{3}$. Both these cases were discussed in the previous sections and both interactions are antiferromagnetic in agreement with the experiments.

We now turn to the two new situations not discussed above. These correspond to the intra-planar exchange in the $a b$ plane, where one of the two Mn atoms has the $d^{4}$ configuration while the other has $d^{3}$. In one case, the two Mn atoms have the " $d^{4}\left(e_{g}\right)-d^{3}$ " configuration in accord with the notation described in Fig. 2. The corresponding Mn-O-Mn triads form the ferromagnetic zigzag chains. In the second case, the two $\mathrm{Mn}$ atoms that belong to two neighboring zigzag chains have the " $d^{4}\left(e_{g}^{\prime}\right)-d^{3}$ ", configuration and are antiferromagnetically coupled. The zigzag chains are shown by heavy lines in Fig. 1(c).

We have obtained the expressions for the exchange interaction in both these cases by using nondegenerate perturbation theory. At first sight, it might appear that the groundstate is degenerate for the present $d^{4}-d^{3}$ situation, since the lone $e_{g}$ electron can be on one of the Mn atoms or the other. This would be true if the two Mn sites were equivalent. However, because the octahedral distortion is present around $\mathrm{Mn}^{3+}$ but not around $\mathrm{Mn}^{4+}$, symmetry is broken and the ground state is in fact nondegenerate. The results of the perturbation theory for the case of the straight bond ( $\theta$ $=180^{\circ}$ ) and neglecting the $t_{2 g}$ hoppings are given by

$$
J^{43}\left(e_{g}-d^{3}\right)=\frac{-t^{4}}{\Delta_{2}^{2}\left(\Delta_{2}+U_{d}+\Delta_{J T}-\Delta_{1}\right)}
$$

for the " $d^{4}\left(e_{g}\right)-d^{3}$ " configuration and

$$
\begin{aligned}
J^{43}\left(e_{g}^{\prime}-d^{3}\right)= & \frac{t^{4}}{16}\left[\frac{-1}{\Delta_{2}^{2}\left(\Delta_{2}+U_{d}+\Delta_{J T}-\Delta_{1}\right)}\right. \\
& +\frac{3}{\Delta_{1}^{2} \Delta_{J T}}+\frac{3\left(\Delta_{1}+\Delta_{2}\right)}{\Delta_{1} \Delta_{2}} \\
& \left.\times\left(\frac{1}{\Delta_{1}\left(\Delta_{1}+\Delta_{2}+U_{p}\right)}+\frac{1}{2 \Delta_{2} \Delta_{J T}}\right)\right]
\end{aligned}
$$

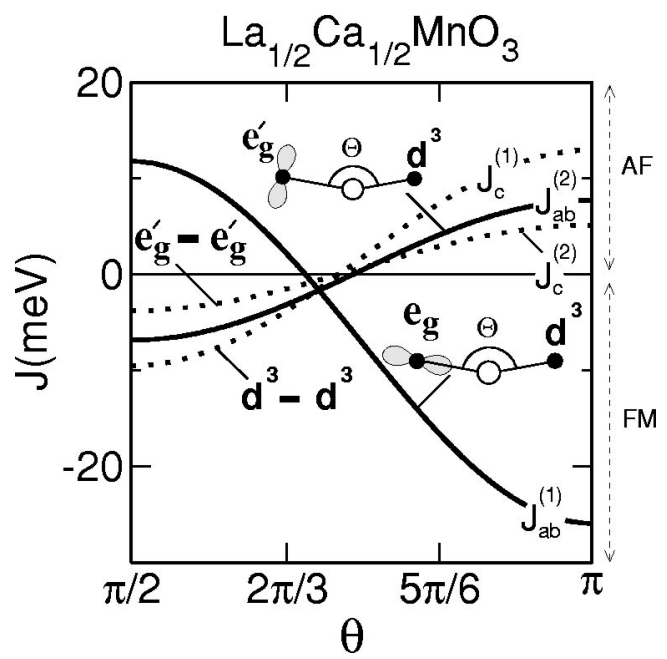

FIG. 11. Calculated exchange for $\mathrm{La}_{1 / 2} \mathrm{Ca}_{1 / 2} \mathrm{MnO}_{3}$ obtained from exact diagonalization of the full Hamiltonian. In the $a b$ plane, there are two different exchange interactions, viz., $J_{a b}^{1}$ corresponding to the $d^{4}\left(e_{g}\right)-d^{3}$ configuration and $J_{a b}^{2}$ corresponding to the $d^{4}\left(e_{g}^{\prime}\right)-d^{3}$ configuration. The exchange interactions $J_{c}^{1}$ and $J_{c}^{2}$ between two Mn atoms along the $c$ axis correspond to the $d^{3}-d^{3}$ and $d^{4}\left(e_{g}^{\prime}\right)-d^{4}\left(e_{g}^{\prime}\right)$ configurations, respectively (see Fig. 1). Results for $J_{c}^{1}$ and $J_{c}^{2}$ are the same as those presented in Figs. 8 and 10.

for the " $d^{4}\left(e_{g}^{\prime}\right)-d^{3}$ " case. Here $\Delta_{1}$ and $\Delta_{2}$ are again the charge transfer energies as defined in Eq. (6). Also, $J_{H}=\infty$ was used to get the above equations.

The ferromagnetic interaction in Eq. (19) comes from the hopping between the $\mathrm{O}\left(p_{z}\right)$ and the $\operatorname{Mn}\left(e_{g}\right)$ orbitals. In Eq. (20), the first term describes the same FM interaction except for the reduced magnitude caused by a lower hopping because of the $e_{g}^{\prime}$ orientation as opposed to the $e_{g}$ orientation in the previous case. The remaining three terms originate from hopping to the $E_{g}^{\prime}$ orbital, which was missing in the previous case because there the $e_{g}^{\prime}$ orbital was involved with zero $\mathrm{O}\left(p_{z}\right)-e_{g}^{\prime}$ hopping. This latter contribution turns out to be antiferromagnetic and it in fact dominates the exchange, producing an overall AF interaction.

In Fig. 11, we show the exchange computed for $\mathrm{La}_{1 / 2} \mathrm{Ca}_{1 / 2} \mathrm{MnO}_{3}$ from exact diagonalization using the full Hamiltonian. These results are consistent with the perturbation theory Eqs. (19) and (20). As seen from the figure, the exchange interaction for the " $d^{4}\left(e_{g}\right)-d^{3}$ " case is ferromagnetic consistent with the ferromagnetic zigzag chains on the $a b$ plane, while it is antiferromagnetic for the " $d^{4}\left(e_{g}^{\prime}\right)-d^{3}$ ", case, consistent with the AF interchain interaction. Thus, all magnetic exchange interactions are correctly described for the $\mathrm{La}_{1 / 2} \mathrm{Ca}_{1 / 2} \mathrm{MnO}_{3}$ compound.

The calculated exchange interaction and the corresponding experimental values for all three manganites are summarized in Table IV. The calculated signs of $J$ 's are robust and agree with experiment in all cases. The calculated magnitudes of $J$ can be brought into perfect agreement with the experiment by taking a slightly different $t$ for each case. 
TABLE IV. Comparison of the calculated $J$ 's with the experimental values in $\mathrm{meV}$. The calculated values are for the parameters of Table II. In $\mathrm{LaMnO}_{3}$, we define $J_{a b}$ and $J_{c}$ as the interplane and intraplane exchange interaction energies. In $\mathrm{La}_{1 / 2} \mathrm{Ca}_{1 / 2} \mathrm{MnO}_{3}$, $J_{a b}^{(1)}$ and $J_{a b}^{(2)}$ are defined respectively as the intrachain and interchain exchange interactions in the $a b$ plane and $J_{c}^{(1)}$ and $J_{c}^{(2)}$ as the $\mathrm{Mn}^{4+}-\mathrm{Mn}^{4+}$ and $\mathrm{Mn}^{3+}-\mathrm{Mn}^{3+}$ exchange interactions in the $c$ direction. The $J$ 's for $\mathrm{La}_{1 / 2} \mathrm{Ca}_{1 / 2} \mathrm{MnO}_{3}$ are not known experimentally except for the signs [Ref. 10]. The $J$ 's in the Table correspond to the $J^{\mu \nu}$, s in the text as follows: $J=J_{c}^{(1)}=J^{33}$, $J_{a b}=J^{44}\left(e_{g}-e_{g}^{\prime}\right), \quad J_{c}=J_{c}^{(2)}=J^{44}\left(e_{g}^{\prime}-e_{g}^{\prime}\right), \quad J_{a b}^{(1)}=J^{43}\left(e_{g}-d^{3}\right), \quad$ and $J_{a b}^{(2)}=J^{43}\left(e_{g}^{\prime}-d^{3}\right)$.

\begin{tabular}{lccc}
\hline \hline & Exchange & Experiment & Theory \\
\hline $\mathrm{CaMnO}_{3}$ & $J$ & 13.1 [Refs. 10,24] & 13.1 \\
$\mathrm{LaMnO}_{3}$ & $J_{a b}$ & -13.4 [Ref. 28] & -15.5 \\
& $J_{c}$ & 9.7 [Ref. 28] & 5.1 \\
$\mathrm{La}_{1 / 2} \mathrm{Ca}_{1 / 2} \mathrm{MnO}_{3}$ & $J_{a b}^{(1)}$ & - & -25.9 \\
& $J_{a b}^{(2)}$ & + & 7.7 \\
& $J_{c}^{(1)}$ & + & 13.1 \\
& $J_{c}^{(2)}$ & + & 5.1 \\
\hline \hline
\end{tabular}

\section{RELATIONSHIP WITH ANDERSON-HASEGAWA DOUBLE EXCHANGE}

In this section, we clarify the relationship between the analysis presented above and the Anderson-Hasegawa double exchange. Recall that in the standard AndersonHasegawa double exchange, the exchange energy goes as $E$ $=-t \cos (\chi / 2)$, where $\chi$ is the angle between the two Mn core spins taken here as classical spins. In the AndersonHasegawa treatment, the $t_{2 g}$ spins are treated as classical, core spins with no $t_{2 g}$ hopping and $J_{H}=\infty$. Our discussion in this section pertains to these approximations for the sake of concreteness.

If one extends the model slightly to allow for the two Mn sites to have different energies (differing by $V$ ), then following the logic of Anderson-Hasegawa, the exchange energy is easily found to be

$$
E(\chi)=-\frac{1}{2} \sqrt{V^{2}+4 t^{2} \cos ^{2}(\chi / 2)}
$$

which goes as $\cos \chi$ instead of $\cos (\chi / 2)$ in the limit $t / V \ll 1$.

Our results for the manganites shows the $J \sim t^{4}$ dependence, even for $\mathrm{Mn}^{3+}-\mathrm{O}-\mathrm{Mn}^{4+}$ where the double-exchange ideas should be appropriate. To clarify the reasons for this, we consider a three-site model keeping only a single nondegenerate orbital on the intermediate atom for simplicity (Fig. 12). The model has three parameters: (i) the Mn-O hopping integral $t$, (ii) the on-site energy difference between the two Mn sites $V$, and (iii) the Mn-O charge-transfer energy $\Delta$. We again take the double-exchange limit $J_{H} \gg t$, so that only one spin state parallel to the classical core spin is accessible.

The Hamiltonian matrix is quite simple since we have just four many-particle configurations: $|1110\rangle,|1101\rangle,|1011\rangle$, and $|0111\rangle$, with the single-particle occupancies in the order $|\mathrm{O} \uparrow\rangle$, $|\mathrm{O} \downarrow\rangle, \mid \mathrm{Mn}($ left $)\rangle$, and $\mid \mathrm{Mn}($ right $)\rangle$. The spins of the Mn sites

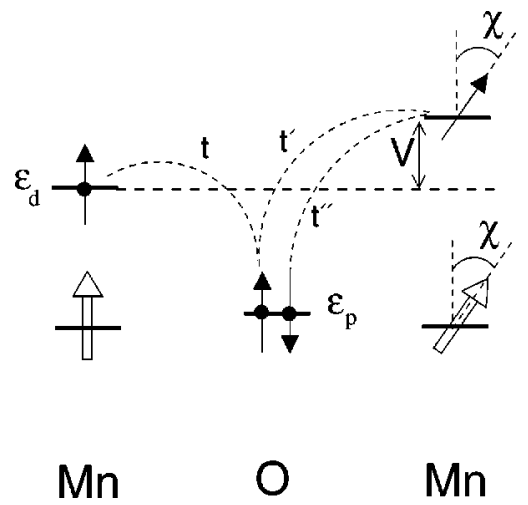

FIG. 12. Three-site model to study the Anderson-Hasegawa double exchange when magnetic interaction is allowed only via hopping to an intermediate atom, with no direct Mn-Mn hopping present. The big arrows indicate the Mn core spins that are treated here as classical spins. Small arrows show the spin orientation of the one-particle states while the dots indicate their occupancies. The hoppings $t^{\prime}$ and $t^{\prime \prime}$ are given by $t^{\prime}=t \cos (\chi / 2)$ and $t^{\prime \prime}=t \sin (\chi / 2)$ following the Anderson-Hasegawa logic.

are fixed by the orientation of the core spins as indicated in Fig. 12. Taking the energy of the first configuration to be $\epsilon$, we have

$$
H(\chi)=\left(\begin{array}{cccc}
\epsilon & 0 & -t \sin (\chi / 2) & t \cos (\chi / 2) \\
0 & \epsilon+V & 0 & -t \\
-t \sin (\chi / 2) & 0 & \epsilon+V+\Delta & 0 \\
t \cos (\chi / 2) & -t & 0 & \epsilon+V+\Delta
\end{array}\right)
$$

The ground-state energy can be obtained by diagonalization, which for $V=0$ is given by

$$
E_{g r}(\chi)=\epsilon+\frac{\Delta}{2}-\sqrt{\frac{\Delta^{2}}{4}+t^{2}(1+\cos (\chi / 2))} .
$$

Retaining terms to the lowest order in $t$, we have

$$
E_{g r}(\chi) \approx \epsilon-\frac{t^{2}}{\Delta}(1+\cos (\chi / 2))
$$

The ground-state energy is of the Anderson-Hasegawa form with the effective hopping $t_{e f f}=t^{2} / \Delta$ as one might have expected since hopping takes place via an intermediate atom. In addition, the $\cos (\chi / 2)$ form is retained.

For $V \neq 0$, it is tedious to write down the exact groundstate energy. It suffices to obtain the perturbation-theory result,

$$
E_{g r}(\chi) \approx \epsilon-\frac{t^{2}}{(V+\Delta)}+\frac{t^{4}}{(V+\Delta)^{3}}-\frac{t^{4}}{2 V(V+\Delta)^{2}}(1+\cos \chi)
$$

Note that the exchange is still ferromagnetic and varies as $t^{4}$ (apart from the constant terms that do not depend on the angle $\chi$ ). In addition we now have the $\cos \chi$ dependence instead of the $\cos (\chi / 2)$ dependence obtained for $V=0$. The 


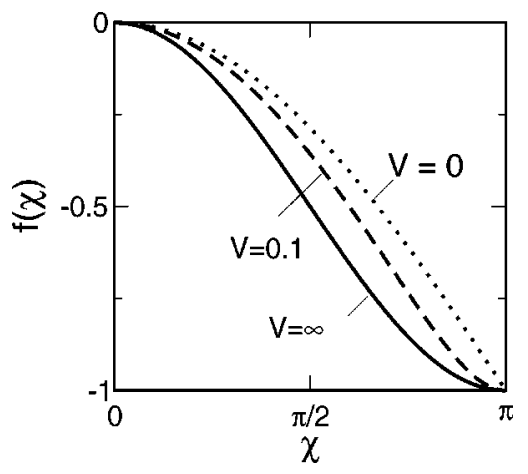

FIG. 13. Dependence of the exchange energy $E(\chi)$ on the relative orientation $\chi$ of the Mn core spins calculated from the diagonalization of Eq. (22) for different values of $V$. Other parameters are $t=1 \mathrm{eV}$ and $\Delta=5 \mathrm{eV}$. Plotted along the $y$ axis is the function $f(\chi) \equiv\left[E(\chi)-E_{\pi}\right] /\left(E_{\pi}-E_{0}\right)$. Note that the angle dependence changes gradually from the Anderson-Hasegawa $\cos (\chi / 2)$ form (dotted line) to the Heisenberg $\cos (\chi)$ form (solid line) as $V$ is varied from 0 to $\infty$.

$t^{4}$ dependence of $J$ for the manganites obtained in the previous sections is similar to what we obtain from Eq. (25). This dependence originates from the fact that the magnetic interaction occurs via an intermediate atom plus the fact that the on-site energies of the two $\mathrm{Mn}$ atoms in $\mathrm{Mn}^{3+}-\mathrm{O}-\mathrm{Mn}^{4+}$ are different on account of the Jahn-Teller distortions of the surrounding octahedra.

The angle dependence of the exchange energy $E(\chi)$ obtained from the diagonalization of Eq. (22) is shown in Fig. 13. The figure shows a crossover between the $\cos \chi / 2$ and the $\cos \chi$ behavior of the exchange energy as obtained from the perturbation results, Eqs. (24) and (25).

\section{CONCLUSION}

In summary, we have studied in detail the magnetic exchange interaction between two $\mathrm{Mn}$ atoms mediated by the oxygen atom on the Mn-O-Mn triad, taking into account the Jahn-Teller induced orbital orientation of the $\operatorname{Mn}\left(e_{g}\right)$ orbitals and the appropriate $\operatorname{Mn}(d)-\mathrm{O}(p)$ hopping. The magnetic exchange depends very strongly on the valence state of the Mn atoms as well as on the $e_{g}$ orbital orientation, in such a way that the magnetic structure of all three manganites, viz., $\mathrm{CaMnO}_{3}, \mathrm{LaMnO}_{3}$, and $\mathrm{La}_{1 / 2} \mathrm{Ca}_{1 / 2} \mathrm{MnO}_{3}$, are explained in a unified manner within our theory. Figures 8, 10, and 11 and Table IV summarize the calculated exchanges for these three compounds.

Listed in Table II are the Hamiltonian parameters used, which were taken with guidance from earlier densityfunctional calculations and photoemission experiments. The sign of the exchange is generally insensitive to the Hamiltonian parameters. However, the magnitude of the exchange has a strong dependence on the electronic hopping $t$, varying as strongly as $t^{4}$ in the fourth-order perturbation theory. In view of this, the magnitude of the hopping was fitted to reproduce the measured exchange for $\mathrm{CaMnO}_{3}$, which yielded a reasonable value for the hopping parameter $V_{d d \sigma}$ $=1.65 \mathrm{eV}$, as listed in Table II. With these parameters, the calculated exchanges for all three compounds along with the experimental values, wherever known, are listed in Table IV.

We have also considered the effect of the $t_{2 g}$ electron hopping on the exchange, an effect often neglected by treating the $t_{2 g}$ spin as a localized $S=3 / 2$ core spin. The effect of retaining the $t_{2 g}$ hopping is to add a ferromagnetic contribution to the exchange, which is substantial for a large deviation of the Mn-O-Mn bond from the linear bond. We have shown how this contribution can change the sign of the exchange interaction as a function of the Mn-O-Mn bond angle $\theta$. In the case of $\mathrm{CaMnO}_{3}$, for instance, the exchange interaction changes from antiferromagnetic to ferromagnetic if $\theta$ is below a critical value $\theta_{c} \leqslant 132^{\circ}$ for the chosen parameters of the Hamiltonian (see Fig. 8). The possibility of such a crossover is long known and recently it has been observed in a somewhat different system $\mathrm{SeCuO}_{3} \cdot{ }^{23}$

The effect of the Hund's energy $J_{H}$ on the exchange was also studied in order to assess the validity of the approximation $J_{H} \rightarrow \infty$, often used in many theoretical works for simplicity. We find that there is a large dependence of the exchange on the magnitude of $J_{H}$. In fact, if the $J_{H} \vec{S} \cdot \vec{\sigma}$ form of the Hund's coupling is used and $t_{2 g}$ hopping is neglected, then in the limit of $J_{H}=0$, magnetic exchange is strictly zero, as the itinerant electrons are not coupled to the $t_{2 g}$ core spins. Equation (11) provides an analytical expression for the $J_{H}$ dependence of the exchange for $\mathrm{CaMnO}_{3}\left(\mathrm{Mn}^{4+}-\mathrm{O}-\mathrm{Mn}^{4+}\right.$ case $)$, obtained using the fourthorder perturbation theory. From this equation as well as from Figs. 7 and 8, where exchange has been plotted for several values of $J_{H}$, it is seen that a finite $J_{H}(\sim 1 \mathrm{eV}$ in typical solids) significantly reduces the magnitude of the exchange as compared to the $J_{H} \rightarrow \infty$ value.

In Sec. IV, we discussed the relationship between the Anderson-Hasegawa double-exchange model and our more elaborate model, showing how the familiar double-exchange form $t_{\text {eff }} \cos (\chi / 2)$ can be recovered from our model as a limit, when one itinerant electron is available to hop between two Mn sites. In the limit that $t<<\Delta$, the effective Anderson-Hasegawa hopping was shown to be given by $t_{\text {eff }}=t^{2} / \Delta$, where $t$ is the Mn-O hopping and $\Delta$ is the Mn-O charge-transfer energy. Another interesting result was the finding that the angle dependence of the exchange interaction changes gradually from the Anderson-Hasegawa $\cos (\chi / 2)$ form to the Heisenberg $\cos (\chi)$ form as the two Mn sites are made unequal by taking a different value for the energy of the itinerant electron (Figs. 12 and 13).

Finally, we note that even though we have focused our attention on the manganites in this paper, the model and the results presented here form a framework for discussion of the magnetic exchange in a variety of other materials as well.

\section{ACKNOWLEDGMENTS}

We thank T. A. Kaplan for stimulating discussions. This work was supported in part by a grant from the Department of Energy under Contract No. DOE FG02-00E0045818. 
*Present address: Information Office, ESRF BP2000, 38043 Grenoble, France.

${ }^{1}$ J. B. Goodenough, J. Phys. Chem. Solids 6, 287 (1958); J. B. Goodenough and A. L. Loeb, Phys. Rev. 98, 391 (1955).

${ }^{2}$ J. Kanamori, J. Phys. Chem. Solids 10, 87 (1959).

${ }^{3}$ P. W. Anderson, Phys. Rev. 115, 2 (1959); in Magnetism I, edited by G. T. Rado and H. Suhl (Academic Press, New York, 1963).

${ }^{4}$ J. B. Goodenough, Magnetic Properties of Perovskites, LandoltBornstein Tabellen, Vol. II, pt. 9 (Springer-Verlag, Berlin, 1962), pp. 2-187.

${ }^{5}$ I. Solovyev, N. Hamada, and K. Terakura, Phys. Rev. Lett. 76, 4825 (1996).

${ }^{6}$ Y.-S. Su, T. A. Kaplan, S. D. Mahanti, and J. F. Harrison, Phys. Rev. B 61, 1324 (2000).

${ }^{7}$ S. Satpathy, Z. S. Popović, and F. R. Vukajlović, Phys. Rev. Lett. 76, 960 (1996); J. Appl. Phys. 79, 4555 (1996).

${ }^{8}$ Z. S. Popović and S. Satpathy, Phys. Rev. Lett. 84, 1603 (2000).

${ }^{9}$ W. E. Pickett and D. J. Singh, Phys. Rev. B 53, 1146 (1996).

${ }^{10}$ E. O. Wollan and W. C. Koehler, Phys. Rev. 100, 545 (1955).

${ }^{11}$ J. B. Goodenough, Phys. Rev. 100, 564 (1955).

${ }^{12} \mathrm{~J}$. B. Goodenough, Magnetism and Chemical Bond (Interscience, New York, 1963).

${ }^{13}$ K. I. Kugel and D. I. Khomskii, Sov. Phys. Usp. 25, 231 (1982).

${ }^{14}$ S. Ishihara, J. Inoue, and S. Maekawa, Phys. Rev. B 55, 8280 (1997).

${ }^{15}$ J. C. Slater and G. F. Koster, Phys. Rev. 94, 1498 (1954).
${ }^{16}$ W. A. Harrison, in Electronic Structure and the Properties of Solids (Freeman, San Francisco, 1980).

${ }^{17}$ A. E. Bocquet, T. Mizokawa, T. Saitoh, H. Namatame, and A. Fujimori, Phys. Rev. B 46, 3771 (1992).

${ }^{18}$ J. H. Jung, K. H. Kim, D. J. Eom, T. W. Noh, E. J. Choi, J. Ju, Y. S. Kwon, and Y. Chung, Phys. Rev. B 55, 15489 (1997).

${ }^{19}$ G.-M. Zhao, K. Conder, H. Keller, and K. A. Müller, Nature (London) 381, 676 (1996); I. Isaac and J. P. Franck, Phys. Rev. B 57, 5602 (1998).

${ }^{20}$ V. Z. Kresin and S. A. Wolf, Philos. Mag. B 76, 241 (1997).

${ }^{21}$ S. Satpathy, J. Phys.: Condens. Matter 10, L501 (1998); H. Meskine and S. Satpathy, J. Appl. Phys. 85, 4346 (1999); S. Satpathy, Solid State Commun. 112, 195 (1999).

${ }^{22}$ A. J. Millis, Phys. Rev. B 55, 6405 (1997).

${ }^{23}$ M. A. Subramanian, A. P. Ramirez, and W. J. Marshall, Phys. Rev. Lett. 82, 1558 (1999).

${ }^{24}$ G. S. Rushbrooke, G. S. Baker, Jr., and P. J. Wood, in Phase Transitions and Critical Phenomena, edited by C. Domb and M. S. Green (Academic Press, London, New York, 1974), Vol. 3, Eqs. (1.1) and (5.4).

${ }^{25}$ P. W. Anderson and H. Hasegawa, Phys. Rev. 100, 675 (1955).

${ }^{26}$ P.-G. de Gennes, Phys. Rev. 118, 141 (1960).

${ }^{27}$ C. Zener, Phys. Rev. 82, 403 (1951).

${ }^{28}$ K. Hirota, N. Kaneko, A. Nishizawa, and Y. Endoh, J. Phys. Soc. Jpn. 65, 3736 (1996). 